\title{
Vestibular Paroxysmia with Trigeminal Neuralgia: Simultaneous Occurrence of Two Compression Syndromes: A Rare Case Report
}

\author{
Anita Bhandari¹, Shivam Sharma ${ }^{2, \odot}$ \\ ${ }^{1}$ Department of Ear, Nose, and Throat, Vertigo and Ear Clinic, \\ Jaipur, Rajasthan, India \\ 2Department of Otorhinolaryngology, Sawai Man Singh Medical \\ College and Hospital, Jaipur, Rajasthan, India
}

\author{
Address for correspondence Shivam Sharma, MS, C-17, Deepak \\ Marg, Adarsh Nagar, Jaipur 302004, Rajasthan, India \\ (e-mail: shivam92@hotmail.com).
}
Abstract
Keywords
- neurovascular compression
- vestibular paroxysmia
- trigeminal neuralgia

Neurovascular compression syndromes are compression of cranial nerves by blood vessels leading to troublesome symptoms. They mostly occur due to a single vessel loop compression. Here, we report two rare cases with simultaneous fifth and eighth nerve compression by anterior inferior cerebellar artery and superior cerebellar artery loops together.

\section{Introduction}

Neurovascular compression syndrome (NVCS) is defined as direct contact of blood vessels causing mechanical irritation of cranial nerves (CNs). ${ }^{1}$ The most common neurovascular compression syndromes are trigeminal neuralgia (TN; compression of CN V), hemifacial spasm (HFS; CN VII), vestibulocochlear paroxysmia (CN VIII), and glossopharyngeal neuralgia (GN; CN IX). Not all cases of NVCS are symptomatic. ${ }^{1}$

Trigeminal neuralgia is a chronic pain disorder of the face with an incidence of 4 to 20 per 100,000. In approximately $80 \%$ of cases, it is caused when the trigeminal nerve is compressed by the superior cerebellar artery (SCA). ${ }^{2}$ Vestibular paroxysmia is a compression syndrome that manifests when arteries at the cerebellopontine angle cause a segmental, pressure-induced dysfunction of the eighth nerve. The symptoms are usually triggered by direct pulsatile compression with ephaptic discharges, less often by conduction blocks. ${ }^{3}$ A loop of the anterior inferior cerebellar artery (AICA) is most often involved, less so the posterior inferior cerebellar artery, the vertebral artery, or a vein. Vestibular paroxysmia (VP) presents as recurrent, spontaneous, short attacks of spinning or nonspinning vertigo that generally last less than 1 minute and can occur in a series of up to 30 or more per day. Attacks

Published online March 27, 2020
DOI https://doi.org/

10.1055/s-0040-1703972.

ISSN 2581-9607. may often be triggered by particular head positions or hyperventilation. Ryu et al have shown that vertigo appears to be associated with vascular compression of the rostroventral nerve (vestibular nerve), while tinnitus appears to be associated with compression of the caudal surface (cochlear nerve) of the nerve. ${ }^{1}$

AICA and SCA loops can be found commonly, but simultaneous occurrence of these two as neurovascular compression syndromes is rare. Here, we report two such cases with symptoms due to vascular loop compression of both the fifth and the eighth nerves, leading to presentation of vestibular paroxysmia with trigeminal neuralgia.

\section{Case Report}

\section{Case 1}

A 61-year-old female patient presented with complaints of approximately 20 to 30 episodes of vertigo with tinnitus each day lasting for approximately 10 seconds since 5 years. She had pain on the right side of her face associated with it and complained of increased vertigo with head movements. The hearing was normal. Videonystagmography (VNG) showed nystagmus on hyperventilation. The MR angiogram showed compression of fifth, seventh, and eighth nerves by loops of SCA and AICA bilaterally. 


\section{Case 2}

A 68-year-old female patient presented with complaints of recurrent episodic vertigo and hearing loss. She also complained of right sided facial pain that occurred in bouts. On vestibular evaluation, the patient was found to have hyperventilation-induced nystagmus on VNG. All other neurootological tests were normal. Audiometry showed a moderate hearing loss on the left side and mild loss on the right. On magnetic resonance imaging (MRI) brain, AICA loop leading to eighth nerve compression on both sides along with a SCA loop compressing upon the fifth nerve on the right side was found.

A diagnosis of vestibular paroxysmia with trigeminal neuralgia was made in both the cases and the patients were put on carbamazepine $100 \mathrm{mg}$ twice a day and were asked to follow-up after 6 weeks (-Figs. 1 and -Figs. 2 ).

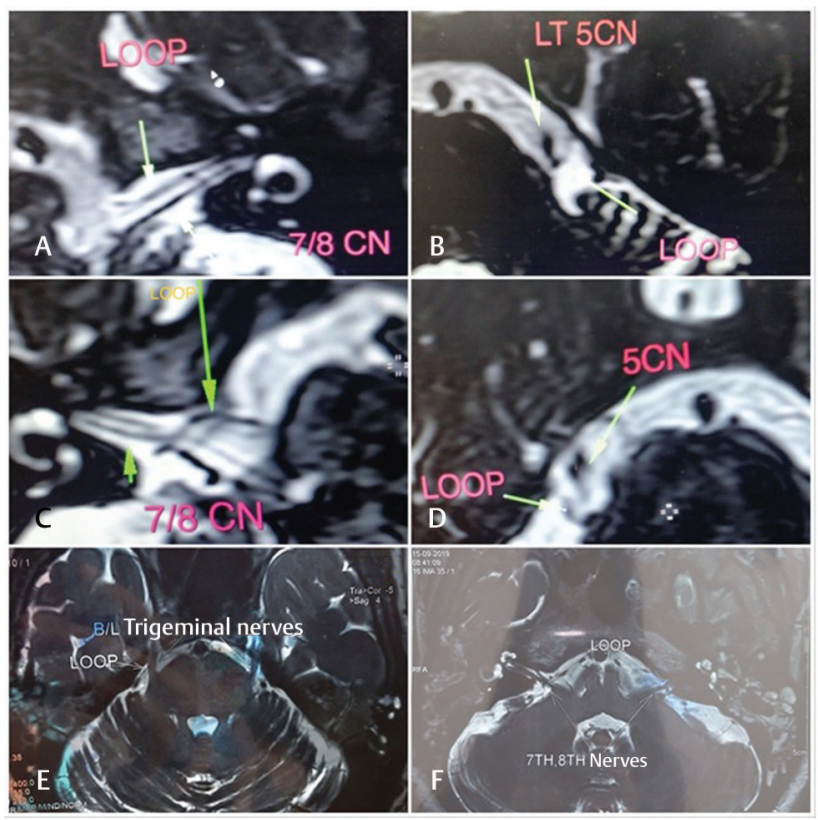

Fig. 1 MRI brain scans of both cases with labeled loops and vessels. (A-D: case 1; E and F: case 2). CN, cranial nerve; MRI, magnetic resonance imaging.

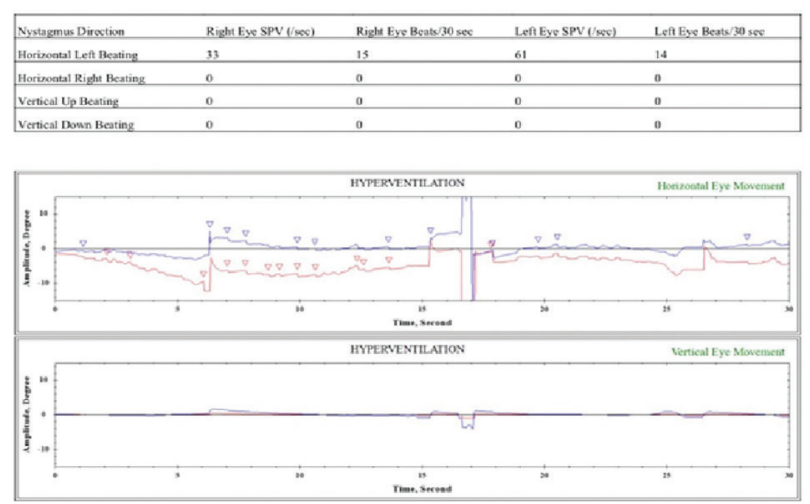

Fig. 2 VNG showing hyperventilation-induced nystagmus. SPV, slow phase velocity; VNG, videonystagmography.

\section{Discussion}

The term vascular compression syndrome, introduced by McKenzie in 1936, ${ }^{4}$ refers to a group of disorders caused by direct contact between a blood vessel and a CN. Jannetta et al suggested that redundant arterial loops could interfere with the vestibulocochlear nerve function resulting in otologic symptoms. ${ }^{5}$ Loops of vessels running near the CNs may be created mostly by degenerative alterations of aging, which can compress surrounding neural elements. ${ }^{6}$

The anatomical variations of the neurovasculature in the cerebellopontine angle result from the late development of the AICA and the posterior inferior cerebellar artery (PICA) from the primitive lateral basivertebral anastomosis. These vascular variations may cause dislocation and compression of the CNs in the posterior cranial fossa. ${ }^{7}$

The Chavda classification grades the vascular loops in the AICA as follows: grade I, when an AICA vascular loop borders the internal auditory meatus; grade II, when the loop insinuates itself into the internal auditory meatus but occupies 50\% or less of the canal; and grade III, when the loop occupies more than $50 \%$ of the canal. ${ }^{4}$

The transition zone between the central and peripheral myelin is the most vulnerable region for symptomatic neurovascular compression syndromes. Trigeminal nerve has a transition zone of $4 \mathrm{~mm}$. Vestibular nerve has a transition zone of $11 \mathrm{~mm}$, with symptomatic neurovascular compression typically at the internal auditory canal. ${ }^{1}$

Vestibular paroxysmia can present as severe vertigo and/ or hearing loss with tinnitus. The most commonly implicated vessel in vestibular paroxysmia is the anterior inferior cerebellar artery (AICA). Many patients develop nystagmus with hyperventilation (and with exercise), because it leads to transient changes in conductivity across the demyelinated portion of the vestibulocochlear nerve causing ephaptic discharges. ${ }^{8}$

NVCS of CN V is usually caused by a neighboring elongated superior cerebellar artery coming from above or by an anterior inferior cerebellar artery (AICA) coming from below, with the superior cerebellar artery being more common (88\% alone or in association) than the AICA ( $\leq 25 \%)$. Both the superior cerebellar artery and AICA usually compress the nerve in its superomedial portion (60\%).

The management starts with low doses of carbamazepine (200-600 mg per day) or oxcarbazepine (300-900 mg per day). These drugs act as blockers of the transmembrane fast sodium channels, thus reducing neuronal excitability. Only in cases refractory to medical treatment, surgical microvascular decompression should be considered. ${ }^{8}$

The simultaneous occurrence of these two neurovascular compression syndromes is a rarity. To the best of our knowledge, only one another such case report has been reported in the literature. Gajski et al published a single case report in 2018, which along with SCA and AICA also had a petrosal vein complex leading to NVCS. ${ }^{2}$

\section{Consent}

Informed consent was obtained from all individual participants included in the study. 


\section{Ethical Approval}

All procedures performed in studies involving human participants were in accordance with the ethical standards of the institutional and national research committee and with the 1964 Helsinki declaration and its later amendments or comparable ethical standards.

\section{Funding}

None.

\section{Conflict of Interest}

None declared.

\section{References}

1 Ryu H, Yamamoto S. Neurovascular decompression of the eighth cranial nerve for intractable vertigo and tinnitus. Operative Techniques in Neurosurgery 2001;4(3):142-152

2 Gajski D, Dennis AR, Arnautović KI. Microsurgical decompression of trigeminal neuralgia caused by simultaneous double arterial (SCA and AICA) and petrosal vein complex compression. J Neurol Surg B Skull Base 2018;79(Suppl 5):S428-S430

3 Brandt T, Strupp M, Dieterich M. Vestibular paroxysmia: a treatable neurovascular cross-compression syndrome. J Neurol 2016;263(Suppl 1):S90-S96

4 de Abreu Junior L, Kuniyoshi CH, Wolosker AB, et al. Vascular loops in the anterior inferior cerebellar artery, as identified by magnetic resonance imaging, and their relationship with otologic symptoms. Radiol Bras 2016;49(5):300-304

5 Jannetta PJ, Møller MB, Møller AR. Disabling positional vertigo. N Engl J Med 1984;310(26):1700-1705

6 Kuncz A, Vörös E, Barzó P. [Vascular compression syndromes of the cranial nerves]. [in Hungarian] Ideggyogy $\mathrm{Sz}$ 2011;64(1-2):6-13

7 Borghei-Razavi H, Darvish O, Schick U. Disabling vertigo and tinnitus caused by intrameatal compression of the anterior inferior cerebellar artery on the vestibulocochlear nerve: a case report, surgical considerations, and review of the literature. J Neurol Surg Rep 2014;75(1):e47-e51

8 Carneiro de Sousa P, Borges da Costa J, Duarte D, Trigueiros N. Vestibular paroxysmia: a diagnosis not to forget. J Otolaryngol 2018;8(2):26-27 